\title{
RINGS IN WHICH THE UNIQUE PRIMARY DECOMPOSITION THEOREM HOLDS
}

\author{
ROBERT W. GILMER
}

Every ring considered in this paper will be assumed to be commutative and to contain an identity element. A ring $R$ will be said to be a $W$-ring (or to have property $W$ ) if each ideal of $R$ may be uniquely represented as an intersection of finitely many primary ideals. A ring $R$ will be called a $W^{*}$-ring (or to have property $W^{*}$ ) if $R$ is a $W$-ring and every ideal of $R$ contains a power of its radical. A quasi-local ring is a ring with exactly one maximal ideal. An integral domain $D$ is strongly integrally closed if it has the following property: If $x$ is an element of the quotient field $K$ of $D$ such that all powers of $x$ are in a finite $D$-module contained in $K$, then $x \in D$.

This paper gives necessary and sufficient conditions that a ring be a $W$-ring. The ideal theory of $W$-rings and $W^{*}$-rings is also investigated. Mori [3] has given necessary and sufficient conditions for a ring to be a $W$-ring, but his characterization of such rings is fairly involved due to the fact that he does not assume his ring has an identity.

The terminology used in this paper is that of Zariski and Samuel [6]. The symbol $\subseteq$ is used to denote containment while $C$ denotes proper containment. Some elementary properties of $W$-rings and $W^{*}$-rings are these:

Property 1. If $R$ is a $W$-ring (respectively, $W^{*}$-ring) and $A$ is an ideal of $R$, then $R / A$ is a $W$-ring ( $W^{*}$-ring) [6, pp. 148-154].

PROPERTy 2. If ring $R$ has property $W$ (respectively, $W^{*}$ ) and $M$ is a multiplicative system of $R$ such that $0 \notin M$, then $R_{M}$, the ring of quotients of $R$ with respect to $M$, has property $W\left(W^{*}\right)$. In particular, if $P$ is a proper prime ideal of $R$, then the quotient ring $R_{P}$ of $R$ with respect to $P$ has property $W\left(W^{*}\right)[6$, p. 225].

Property 3. A finite direct sum of rings is a $W$-ring ( $W^{*}$-ring) if and only if each summand is a $W$-ring ( $W^{*}$-ring) [6, p. 175].

LemMa 1. If $R$ is a quasi-local ring having property $W, R$ is a primary ring or a one-dimensional integral domain.

Proof. We suppose that $R$ is not a primary ring. Then $R$ is not zero-dimensional. We shall first show that $R$ is one-dimensional. Suppose that there exist proper prime ideals $P_{0}, P_{1}, P_{2}$ of $R$ such that $P_{0} \subset P_{1} \subset P_{2}$. By passage to $R / P_{0}$ we may suppose $R$ is a $W$-domain

Received by the editors March 22, 1962 and, in revised form, July 5, 1962. 
containing a nonmaximal prime ideal $P \neq(0)$. We choose $p \in R \backslash P$ such that $P+(p)$ is a proper ideal of $R$. If $M$ is an isolated prime ideal of $P+(p)$, then by Property 3 , we may assume $M$ is the maximal ideal of $R$. $R$ is an integral domain so that if $d \in P, d \neq 0$, then $(d) M$ $C(d)$. Hence there exists an ideal $Q$ primary for a prime ideal $P^{\prime}$ in the primary representation of $(d) M$ such that $d \notin Q$. Since $d M \subseteq Q$, $M \subseteq P^{\prime}$ so that $P^{\prime}=M$ since $M$ is maximal. We have $P \Phi Q$ and $Q \nsubseteq P$. Property 1 implies that we may assume $P \cap Q=(0)$. Further, if $Q_{\alpha}$ is any ideal of $R$ primary for $M$, then $Q \cap Q_{\alpha}=Q$ and hence $Q \subseteq Q_{\alpha}$ from the uniqueness of the representation. Clearly $P$ is the unique minimal prime ideal of $R$. Since $M$ is a minimal prime ideal of $P+(p), \sqrt{ }(p)=M$ so that $(p)$ is primary for $M[6, p .153]$. For some integer $n,\left(p^{n}\right)=(p)^{n} \subseteq Q$. From the minimality of $Q$ among those ideals primary for $M$, we have $Q=(p)^{n}=(p)^{n+1}=\cdots$, a contradiction. Thus $R$ has dimension one.

Now let $M$ be the maximal ideal of $R$ and let $P$ be a nonmaximal prime ideal of $R$. We first show that if $Q$ is primary for $M$, then $P \subset Q$. If this were not the case, then as above we may assume $P \cap Q=(0)$. From the one-dimensionality of $R, P$ and $M$ are the only two prime ideals of $R$. Hence if $q \in Q, q \in P,(q)$ is primary for $M$ so that $Q=(q)=\left(q^{2}\right)$, again a contradiction. We proceed to show that $P=(0)$. By what we have just shown, if $p \in P$ and $p$ is in no other minimal prime ideal of $R$, then $(p)$ is $P$-primary. Also, if $m \in M$ and $m$ is in no minimal prime ideal of $R,(m)$ is $M$-primary. Hence $(p) \subseteq P \subseteq(m)$. Then for some $y \in R, p=m y$. Since $(p)$ is $P$-primary and $m \notin P, y \in(p)$ so that $p=m s p$. Now $1-s m$ is a unit in $R$ so that $p=0$. Therefore $P=(0)$ and $R$ is a domain as asserted.

ThEOREM 1. A ring is a $W$-ring if and only if it is a finite direct sum of primary rings and one-dimensional integral domains in which every nonzero ideal is contained in only finitely many maximal ideals.

Proof. We first prove that a one-dimensional integral domain $J$ in which every nonzero ideal has only finitely many maximal ideal divisors is a $W$-domain. Thus if $A$ is a nonzero ideal of $J$ and if $M_{1}, M_{2}, \cdots, M_{t}$ is the set of maximal ideals containing $A$, we let $Q_{i}$ be the $M_{i}$-primary ideal $J \cap A J_{M_{i}}$. Clearly $A \subseteq B=Q_{1} \cap \cdots \cap Q_{t}$. But for any maximal ideal $M$ of $J, A J_{M}=B J_{M}$ so that $A=B$. The uniqueness of the representation follows easily from the fact that $J$ is one-dimensional [5, p. 45]. Thus by Property 3 , it is clear that the condition given is sufficient.

We now suppose that $R$ is a $W$-ring and $(0)=Q_{1} \cap \cdots \cap Q_{n} \cap P_{1}$ $\cap \cdots \cap P_{m}$ is the primary representation of $(0)$ where each $Q_{i}$ is 
primary for a maximal ideal $M_{i}$ and each $P_{j}$ is primary for a nonmaximal prime ideal. If $M$ is any maximal ideal properly containing some $P_{j}, R_{M}$ is a quasi-local $W$-ring which is not a primary ring. Lemma 1 implies that $P_{j}$ is its own prime radical, that $P_{j}$ is the only prime ideal contained in $M$, and that $P_{j}$ is contained in every $M$ primary ideal of $R$. Thus if $i \neq j, P_{i}$ and $P_{j}$ are comaximal and since the given representation is irredundant, $P_{j}$ and $Q_{k}$ are comaximal for each $k$. Hence $R \simeq R / Q_{1} \oplus \cdots \oplus R / Q_{n} \oplus R / P_{1} \oplus \cdots \oplus R / P_{m} \quad[6$, p. 178]. Clearly each $R / Q_{i}$ is a primary ring and as noted above, each $R / P_{j}$ is a one-dimensional integral domain. Because $R / P_{j}$ is a $W$ domain, every nonzero ideal of $R / P_{j}$ has only finitely many maximal ideal divisors.

TheOREM 2. $A W$-ring is a $W^{*}$-ring if and only if every nonzero ideal of $R$ contains a product of nonzero prime ideals.

Proof. The condition is obviously necessary. In view of Theorem 1 and Property 3, to show the condition is sufficient we need only consider the case when $R$ is a primary $W$-ring or when $R$ is a $W$-domain. In the second case, if $Q \neq(0)$ is a primary ideal of $R$ with radical $P$ and if $Q \supseteq M_{1}^{e_{1}} \cdots M_{t}^{e_{t}}$ where each $M_{i}$ is a maximal ideal of $R$, then $P=M_{i}$ for some $i$ from the one-dimensionality of $R$. If $P=M_{1}$, then $M_{2}^{e_{2}} \cdots M_{t}^{e_{t}} \nsubseteq P$ so that $M_{1}^{e_{1}} \subseteq Q$ and $R$ is a $W^{*}$-domain. If $R$ is a primary $W$-ring with maximal ideal $M$ and if $m \in M, m \neq 0$, then $(m) \supseteq M^{i}$ for some $i$. Thus $(0)=\left(m^{k}\right) \supseteq M^{i_{k}}$ for some $k$. Therefore $R$ is a $W^{*}$-ring.

Combining Theorem 1 with Theorem 2 we obtain:

TheOREM 3. A ring $R^{*}$ is a $W^{*}$-ring if and only if $R^{*}$ is a finite direct sum of primary rings having nilpotent maximal ideals and one-dimensional integral domains in which every nonzero ideal contains a product of nonzero prime ideals.

Corollary 3.1. If $A$ is a proper ideal in a $W^{*}$-ring $R^{*}$, then $\cap A^{n}$ is the intersection of the primary components of (0) contained in $A$.

Proof. In view of Theorem 3 and the obvious validity of the theorem for primary $W^{*}$-rings, it suffices to prove the theorem in the case when $R^{*}$ is a domain and $A$ is a prime ideal, and even in this case we may assume that $R^{*}$ is quasi-local and $A$ its maximal ideal. Then if $a \in A, a \neq 0$, then $\left(a^{2}\right)$ is $A$-primary and $a \notin\left(a^{2}\right)$. Since $\left(a^{2}\right)$ contains a power of $A, a \notin \cap A^{n}$ so that $\cap A^{n}=(0)$ and our proof is complete.

CoRollary 3.2. In a $W^{*}$-domain $J^{*}$ every proper ideal is a product of nonfactorable ideals. 
Proof. Suppose $A, \neq(0)$ or $J^{*}$, is not a product of nonfactorable ideals. Let $P_{1}, P_{2}, \cdots, P_{t}$ be the maximal ideals containing $A$. For any integer $n$ we have $A=A_{1} A_{2} \cdots A_{n t}$ where the $A_{j}$ are proper ideals. Each $A_{j}$ is contained in one of $P_{1}, \cdots, P_{t}$ so that for some $i$, $A \subseteq P_{i}^{n}$. Therefore for some $k, A \subseteq \cap P_{k}^{n}$, a contradiction since $\cap P_{k}^{n}$ $=(0)$.

It is clear that a $W^{*}$-domain $J^{*}$ is a Dedekind domain if and only if primary ideals of $J^{*}$ are prime powers. Or, stated otherwise, $J^{*}$ is a Dedekind domain if and only if $J_{P}^{*}$ is a discrete valuation ring for every prime ideal $P$ of $J^{*}$. However, it is easily seen that if $J_{P}^{*}$ is a valuation ring at all it is discrete [1]. From this observation, it follows that a $W^{*}$-domain satisfying any one of seven conditions of Cohen [2] is a Dedekind domain.

In the following theorem, this result will be used: If $A$ and $B$ are ideals of a $W^{*}$-domain, $A: B=A$ if and only if $B$ is contained in no prime ideal of $A[5$, p. 36].

THEOREM 4. If the $W^{*}$-domain $D$ is strongly integrally closed, then $D$ is a Dedekind domain.

Proof. To show $D$ is Dedekind, it suffices to show that if $P \neq(0)$ is a prime ideal of $D$, then $P$ is invertible [4]. $D$ is one-dimensional so that if $p \in P, p \neq 0, P$ is a prime ideal belonging to $(p)$. Hence $(p): P \supset(p)$. Let $a \in(p): P, a \notin(p)$. If $P^{-1}$ is the set of all elements $s$ of $K$ such that $s P \subseteq D$, then $a / p \in P^{-1}, a / p \notin D$. Now $P \subseteq P P^{-1} \subseteq D$ so that either $P=P P^{-1}=\left(P P^{-1}\right) P^{-1}=\cdots$ or $P$ is invertible. If $P=P\left(P^{-1}\right)^{k}$ for each positive integer $k$, and if $t \in P^{-1}$, all powers of $t$ are in the finite $D$-submodule of $K$ generated by $1 / p$. Since $D$ is strongly integrally closed, $t \in D$ so that $P^{-1} \subseteq D$, a contradiction. Hence $P$ is invertible.

The following example of Krull demonstrates that Theorem 4 need not be valid if $D$ is integrally closed in the ordinary sense. It also shows that a quasi-local $W^{*}$-domain need not be Noetherian.

Let $K$ be a field and let $D$ be the set of all elements $f(x, y) / g(x, y)$ such that $x$ does not divide $g$ and $f(0, y) / g(0, y) \in K$. It is readily checked that $D$ is a quasi-local domain of dimension one. (The maximal ideal, $M$, consists of all $f / g$ where $x$ divides $f$.) If $f / g \in M$ and $n$ is the highest power of $x$ that divides $f$, then $M^{n+1} \subseteq(f / g)$. Hence $D$ is a $W^{*}$-domain. It is easy to check that $D$ is integrally closed. But $D$ is not a valuation ring: let $u=x y / y+1, v=x(y+1) / y$. Neither $u / v$ or $v / u$ lies in $D$. Now if $a_{i}=x(y+1) / y^{i}$ and if $A_{i}=\left(a_{1}, a_{2}, \cdots, a_{i}\right)$, it may be shown that $A_{1} \subset A_{2} \subset \cdots \subset A_{n} \subset A_{n+1} \subset \cdots$, so that $D$ is not Noetherian. 


\section{BIBLIOGRAPHY}

1. Keizô Asano, Über kommutative Ringe, in denen jedes Ideal als Produkt von Primidealen darstellbar ist, J. Math. Soc. Japan 3 (1951), 82-90.

2. I. S. Cohen, Commutative rings with restricted minimum condition, Duke Math. J. 17 (1950), 27-42.

3. Shinziro Mori, Ueber eindeutige Reduktion von Idealen in Ringen ohne Teilerkettensatz, Hiroshima J. Sci. 3 (1933), 275-318.

4. Noburu Nakano, Über die Umkehrbarkeit der Ideale im Integritätsbereiche, Proc. Imp. Acad. Tokyo 19 (1943), 230-234.

5. B. L. van der Waerden, Modern algebra, Vol. II, 2nd ed., Ungar, New York, 1950.

6. Oscar Zariski and Pierre Samuel, Commutative algebra, Vol. I, The University Series in Higher Mathematics, Van Nostrand, Princeton, N. J., 1958.

Louisiana State University 